\title{
Febrile Neutropenia in Patients with Acute \\ Leukemia with Long-Term Central Venous Access in Kuwait: Microbial Spectrum, Outcome and Catheter Management
}

\author{
Soad Al Bahar Ramesh Pandita Ketan Bavishi Bipin Savani \\ Department of Haematology, Kuwait Cancer Control Centre, Kuwait
}

\section{Key Words}

Hickman catheter . Acute leukemia .

Antimicrobial therapy · Infection •

Neutropenia

\begin{abstract}
Objective: This study was conducted to investigate the microbial spectrum, outcome and catheter management in febrile neutropenic acute leukemia patients with long-term central venous access, at a single center in Kuwait. Methods: One hundred and thirtythree febrile neutropenic episodes in 64 adult acute leukemia patients with long-term central venous access encountered at the Kuwait Cancer Control Centre were studied. The frequency of clinically documented infections, microbiologically documented infections, fevers of unknown origin and catheter-related infection was determined. Response to treatment and the need for catheter removal were studied. Results: In the 133 febrile neutropenic episodes, clinically documented infec-
\end{abstract}

\begin{tabular}{ll}
\hline KARGER & ( 2000 S. Karger AG, Basel \\
Fax +4161306 1234 34 1011-7571/00/0091-0035\$17.50/0 \\
$\begin{array}{l}\text { E-Mail karger@karger.ch } \\
\text { www.karger.com }\end{array}$ & $\begin{array}{l}\text { Accessible online at: } \\
\text { www.karger.com/journals/mpp }\end{array}$
\end{tabular}

tions, microbiologically documented infections and fever of unknown origin occurred in $12.8,30.8$ and $56.4 \%$, respectively. Thirtytwo episodes of catheter-related infections were encountered. Gram-positive and gramnegative infections occurred in equal frequency. Escherichia coli and methicillin-resistant Staphylococcus aureus were the most frequent organisms. Of all episodes, 87.2\% responded to antibiotics and 11 episodes required removal of the catheter. Of the clinically documented infections, $76.5 \%$ of the episodes responded to treatment without catheter removal. Of the microbiologically documented episodes, $68.3 \%$ were treated successfully without removal of the catheter. One hundred percent of the episodes of fever of unknown origin responded to a broad spectrum of antibiotics, without catheter removal. Conclusion: Gram-negative and gram-positive infections occur in equal frequency in febrile neutropenic acute leukemia patients with long-term central venous catheters. E. coli and methicillin-resistant $S$. au-
Dr. Soad Al Bahar
Department of Haematology
Kuwait Cancer Control Centre
PO Box 12391

Al Shamiyah 71654 (Kuwait) 
reus are the most frequent organisms and the majority of the episodes are curable by antimicrobial therapy without the removal of the catheter.

Copyright $\odot 2000$ S. Karger AG, Basel

\section{Introduction}

Neutropenia is a major risk factor for infections in leukemic patients. Long-term central venous catheters $(\mathrm{CVC})$ provide a reliable access for the administration of chemotherapeutic agents, blood component transfusions, parenteral nutrition, antibiotics and for drawing blood samples for investigations. However, the CVC is also an important cause of nosocomial blood stream infections. Infections are caused due to the device or at the exit site and occur in $11-34 \%$ of the patients [1]. Among oncology patients, Hickman-Broviac type catheters seem to have lower per day infection rates than peripheral catheters [2] and are advocated as the best choice for longterm venous access [3]. The incidence of CVC-related infections is mainly dependent on the duration of implantation, the underlying disease and the type of catheter [4]. Neutropenia is a major risk factor for catheterrelated infections [5]. The etiopathogenesis of CVC-related infections is multifactorial and causes include contamination of the hub, contamination from the cutaneous entry site and endogenous colonization of the catheter [6]. The organisms most commonly responsible for catheter-based septicemia are coagulasenegative staphylococci and account for $40 \%$ of all isolates [7].

In this study, we report on the microbiological spectrum, outcome and catheter-related infections and catheter management in febrile neutropenic adult leukemic patients with long-term central venous access.

\section{Material and Methods}

Febrile neutropenic episodes in 64 consecutive acute leukemic patients with indwelling Hickman catheters seen at the Kuwait Cancer Control Centre were studied retrospectively. The inclusion criteria were (a) underlying acute leukemia either de novo, in complete remission or in relapse, (b) absolute neutrophil count below $1,000 \times 10^{6} / 1$, (c) fever of $38.5^{\circ} \mathrm{C}$ once or more than $38^{\circ} \mathrm{C}$ on two or more occasions during $12 \mathrm{~h},(\mathrm{~d})$ an indwelling Hickman catheter.

Infections were classified as (a) clinically documented infection, (b) microbiologically documented infection with or without bacteremia, (c) fever of unknown origin when there was no clinical or microbiological documentation but the clinical course was compatible with infection. Catheter-related infections were defined as any of the following: (I) exit site infection: development of erythema, tenderness and induration and or purulence within $2 \mathrm{~cm}$ of the skin exit site of the catheter; (II) tunnel infection: development of erythema, tenderness and induration along the subcutaneous tract ('tunnel') of the Hickman catheter at a distance of more than $2 \mathrm{~cm}$ from the skin exit site with or without signs of inflammation or purulence at the exit site; insertion site infections were included in this category; (III) catheter-related blood stream infection: isolation of the same microorganism from the culture of the blood drawn from the catheter and peripheral vein in the absence of any other local infection caused by the same organism; (IV) septic thrombophlebitis: septic venous occlusion in proximity to the Hickman catheter associated with bacteremia and fever.

The history of all the patients was taken. A complete physical examination, complete blood counts, biochemical renal and liver function tests and urine analysis were carried out. Cultures from the peripheral venous blood, Hickman catheters, urine, throat, stool and sputum were also obtained. The studies were repeated at least twice a week or more frequently if indicated. A chest X-ray was done in all cases and sonographic/computerized axial tomography studies were done as clinically indicated. All patients received initial empiric antibiotic therapy consisting either of antipseudomonal antibiotic + aminoglycoside or antipseudomonal antibiotic + glycopeptide antibiotics. The antibiotic therapy was modified according to the culture/sensitivity reports and empirically in those not responding after $72 \mathrm{~h}$. Parenteral amphotericin B was administered if fever persisted beyond 5 days when on the antibiotics or if there was any microbiological evidence or clinical suspicion of invasive fungal infection. In some patients who were allergic to these antibiotics, 
Table 1. Patient characteristics

\begin{tabular}{lc}
\hline Number of patients/catheters & $64 / 66$ \\
Sex & \\
$\quad$ Male & 44 \\
$\quad$ Female & 22 \\
Age, years & \\
$\quad$ Median & 31 \\
$\quad$ Range & $15-68$ \\
Diagnosis & \\
$\quad$ Acute myeloblastic leukemia & 49 \\
$\quad$ Acute lymphoblastic leukemia & 13 \\
$\quad$ Chronic myeloid leukemia in blast crisis & 1 \\
$\quad$ Myelodysplastic syndrome & 3 \\
Catheter days & \\
$\quad$ Total & 6,670 \\
$\quad$ Mean & 101 \\
\hline
\end{tabular}

other antibiotics were used. The CVC were removed for infectious reasons if bacteremia persisted after $72 \mathrm{~h}$ despite appropriate antibiotic therapy, evidence of nonresolving or progressive local infection despite adequate antibiotic treatment.

\section{Results}

Sixty-four patients with 66 double-lumen Hickman catheters were enrolled in the study. Patient characteristics are given in table 1 . Their ages ranged from 15 to 68 years (median 31 years). The catheters were followed for a total of 6,670 catheter days (mean 101.06 days) until catheter removal or death.

One hundred and thirty-three febrile neutropenic episodes occurred during the 6,670 catheter days (table 2). One hundred episodes were associated with absolute neutrophil counts of less than $100 \times 10^{6} / 1(75.2 \%), 24$ episodes with $100-500 \times 10^{6 / 1}(18 \%)$ and 9 episodes were associated with absolute neutrophil counts of 500-1,000 $\times 10^{6} / 1$. Clinically documented infection occurred in $12.8 \%$ of the episodes and consisted of 5 pneumonias, 7 perianal or cutaneous abscesses, 4 catheter
Table 2. Details of febrile neutropenia episodes

$\begin{array}{lc}\text { Total febrile neutropenia episodes } & 133 \\ \text { Absolute neutrophil count } & \\ \quad<100 \times 10^{6} / 1 & 100(75.2 \%) \\ 100-499 \times 10^{6} / 1 & 24(18.0 \%) \\ 500-1,000 \times 10^{6} / 1 & 9(6.8 \%) \\ \text { Clinically documented infection } & 17 / 133(12.8 \%) \\ \text { Microbiologically documented } & \\ \quad \text { infection } & 41 / 133(30.8 \%) \\ \text { Fever of unknown origin } & 75 / 133(56.4 \%)\end{array}$

Table 3. Febrile neutropenia: organisms isolated

\begin{tabular}{lc}
\hline Microbiologically documented infections & 41 \\
Gram-positive & 20 \\
Methicillin-resistant Staphylococcus aureus & $5(3)$ \\
Staphylococcus epidermidis & $3(3)$ \\
Staphylococcus simulans $^{1}$ & $3(3)$ \\
Staphylococcus sciuri & $1(1)$ \\
Staphylococcus haemolyticus & $1(1)$ \\
Streptococcus group D & $3(1)$ \\
Streptococcus viridans & $2(2)$ \\
Streptococcus mitis & $2(2)$ \\
Gram negative & 20 \\
Escherichia coli & $9(8)$ \\
Pseudomonas aeruginosa & $4(2)$ \\
Acinetobacter calcoaceticus & $3(3)$ \\
Klebsiella pneumoniae & $1(1)$ \\
Pseudomonas paucimobilis ${ }^{1}$ & $1(1)$ \\
Enterobacter cloacae & $1(0)$ \\
Burkholderia cepacia & $1(1)$ \\
Fungal & 2 \\
Candida albicans & $2(2)$
\end{tabular}

Figures in parentheses indicate septicemia. 1 Polymicrobial infections.

exit site infections and 1 chicken pox. Microbiologically documented infections occurred in $30.8 \%$ episodes and were divided into bacteremic and nonbacteremic episodes. The microbial spectrum is given in table 3. Gramnegative and gram-positive organisms were 
Table 4. Microbiological spectrum of catheter-associated infection

\begin{tabular}{lc}
\hline Total & 28 \\
Gram-positive & 13 \\
Methicillin-resistant Staphylococcus aureus & $4(2)$ \\
Streptococcus viridans & $2(2)$ \\
Streptococcus mitis & $2(2)$ \\
Staphylococcus epidermidis & $2(2)$ \\
Staphylococcus simulans ${ }^{1}$ & $2(2)$ \\
Staphylococcus haemolyticus & $1(1)$ \\
Gram-negative & 14 \\
Escherichia coli & $5(5)$ \\
Acinetobacter calcoaceticus & $3(3)$ \\
Pseudomonas aeruginosa & $3(2)$ \\
Burkholderia cepacia & $1(1)$ \\
Klebsiella pneumoniae & $1(1)$ \\
Pseudomonas paucimobilis ${ }^{1}$ & $1(1)$ \\
Fungal & 2 \\
Candida albicans & $2(2)$
\end{tabular}

Figures in parentheses indicate septicemia. 1 Polymicrobial infections.

isolated in equal frequency while polymicrobial organisms and Candida albicans were isolated in 1 and 2 cases, respectively. Amongst the gram-negative infections, Escherichia coli was the most frequent organism (9 episodes) followed by Pseudomonas aeruginosa (4 episodes). In gram-positive infections, methicillin-resistant Staphylococcus aureus was the most frequent isolate (5 episodes); however, other staphylococci and streptococci were also isolated.

Catheter-related infections occurred in $24 \%(32 / 133)$ of the episodes during the 6,670 days of catheter access, which yields a catheter infection rate of $0.48 / 100$ access days. The 32 episodes occurred in 29 patients and consisted of 15 bacteremias, 12 exit site infections, 4 tunnel infections and 1 episode of septic thrombophlebitis.

Twenty-eight out of 32 episodes of catheter-related infections were microbiologically documented and 4 episodes were clinically documented (table 4). Gram-negative organisms were isolated in $46.4 \%$ episodes and $E$. coli was the most frequent organism. Grampositive organisms were isolated in $42.8 \%$ episodes and methicillin-resistant $S$. aureus was the most frequent single organism. Two episodes (7\%) were associated with C. albicans fungemia and 1 with polymicrobial organisms.

The Hickman catheter was removed in 11 out of 133 febrile episodes, involving tunnel infections (3), exit site infections (5/12), bacteremia (2/15) and septic phlebitis (1/1).

Forty-four episodes were treated with ceftazidime + amikacin and $24(54.5 \%)$ responded without modification. Ceftazidime + vancomycin were used in 48 episodes with a response without modification in 29 episodes $(60.4 \%)$. Piperacillin + amikacin were used in 26 episodes, and $8(30.7 \%)$ responded without modification. Other antibiotics were used in 15 episodes with a response in 10 . Amphotericin and vancomycin were the most frequent modifications. A total of 10 patients died, 4 due to infection. Of the 4 that died due to infection, 2 had $C$. albicans septicemia and 2 pneumonia. Two patients died because of progressive disease and infection, 3 patients due to refractory/relapsing disease and 1 patient died of sudden cardiac arrest. Of all episodes, $87.2 \%$ responded to antibiotics and 11 episodes required removal of the catheter. Of the clinically documented infections, $76.5 \%$ of the episodes responded to treatment without catheter removal. Of the microbiologically documented episodes $68.3 \%$ were treated successfully without removal of the catheter. One hundred percent of the episodes of fever of unknown origin responded to a broad spectrum of antibiotics, without catheter removal. Thirty out of 32 episodes of catheter-related infections responded to antimicrobial therapy. The 11 episodes that required removal of 
the Hickman catheter, and the 2 patients who died of $C$. albicans septicemia, showed catheter-related infections.

\section{Discussion}

The Hickman catheter has become an integral part of the management of patients with hematological malignancies [8]. It provides continuous venous access facilitating the administration of drugs and blood components and is highly acceptable to the patients [9]. However, infection remains a major complication of this device. Most patients with acute leukemia develop infection during the neutropenic phase following chemotherapy. The incidence of bacteremia has not changed since the introduction of the Hickman line [3]. In the present study the febrile neutropenic episodes consisted of $12.8 \%$ of clinically documented infection, $30.8 \%$ microbiologically documented infections and $56.4 \%$ fever of unknown origin. This occurrence is similar to that reported by Behre et al. [10] consisting of episodes in patients with and without central venous access. However in large trials, clinically defined infection was reported in $26 \%$ of the episodes [11]. In the present study, $52 \%$ of febrile neutropenic episodes responded to primary antibiotics without modification, and this is in agreement with the experience reported in large series [11]. The reported incidence of the CVC-related infections varies from 8 to $47 \%$ [12]. The variation is due mainly to the different definitions of CVCrelated infection. Our Hickman catheter infection rate of $24 \%$ and $0.48 / 100$ access days was higher when compared to that of $0.14 / 100$ and $0.16 / 100$ catheter access days reported by Press et al. [1] and Mueller et al. [13], respectively. However, others have reported a comparable incidence [12, 14-17]. The higher infection rate in our series may be due to a high proportion of severely neutropenic patients $(75.2 \%)$ during febrile episodes. Neutropenia is a major risk factor for CVC infections [5]. The other possible reason may be the use of double-lumen Hickman catheters in all our patients. Keung et al. [12] have reported a higher infection rate in the multilumen catheters; however, it was not statistically significant. Multilumen catheters are given to more manipulations and have more potential portals of bacterial entry.

In our study, $46.4 \%$ episodes of catheterrelated infections were caused by gram-negative infections and $E$. coli was the most frequent organism. This contrasts with other studies which have reported a higher incidence of gram-positive infections due to $S$. aureus and other gram-positive organisms [1, $7,13,15]$. However, predominant gram-negative infections have been reported by Alurkar et al. [14] and Reilly et al. [18].

Earlier studies have reported an incidence of Hickman catheter exit infection of $40 \%$, tunnel infection of $25 \%$ and septicemic infection of $30 \%$ in cancer patients $[1,13,19]$. We have found exit site infection in $36 \%$, tunnel infection in $12.5 \%$, septicemia in $46 \%$ and septic thrombophlebitis in 5.5\% of the episodes. Although tunnel infection was less frequent, the septicemia rate was higher. We may have overestimated the incidence of Hickman-catheter-related septicemia and some of these infections may have been due to an invasive mucosal organism. Quantitative microbiological colony cultures of blood specimens taken from peripheral veins and Hickman catheters would probably give a more accurate assessment of the incidence of catheter-related bacteremias [5, 20-22].

In our study of 133 febrile episodes, in 11 episodes the catheter was removed, 6 episodes were fatal with the catheter in situ, and the catheter removal rate was $8.2 \%$, a rate comparable with those of other studies [12, 14, 15]. 
We encountered tunnel infection in 4 episodes. In 3 episodes of tunnel infection, the catheter was removed and 1 patient died before the catheter could be removed. In other reported series, tunnel infection required removal of the catheter in most of the instances $[1,14,15,21]$. Measures to reduce the incidence of tunnel infection have included prophylactic glycopeptide administration before the insertion of a Hickman catheter [20].

Exit site infections and bacteremia can be treated with antibiotics in most of the instances without catheter removal. In our patients, catheter removal was required in microbiologically documented infections only. A similar experience has been reported by Riikonen et al. [15]. Patients who remained febrile and in whom blood cultures were negative or became negative, a wait-and-watch policy regarding catheter removal was adopted for 10-14 days. This policy resulted in a favorable outcome in our patients without an increase in mortality or catheter-related deaths. Our data indicate that in leukemia patients with Hickman catheters, $87.2 \%$ of all episodes of febrile neutropenias and $68.3 \%$ of microbiologically documented febrile episodes were curable without the removal of the catheter. Others have reported similar experiences [13,15]. Although no febrile episodes of unknown origin required catheter removal, $31.7 \%$ microbiologically documented episodes responded only after the removal of the catheter. The majority of the patients (118 episodes) were treated initially with a combination of antibiotic therapy consisting of an antipseudomonal antibiotic with either aminoglycoside or glycopeptide with a response rate of $52 \%$ without any modification. In the remaining 15 episodes when other antibiotics were used predominantly because of hypersensitivity, a similar response was obtained. In our study, the major exceptions to the attempt to treat catheter-associated infections without removal of the device were infections caused by Pseudomonas and methicillin-resistant $S$. aureus, which are difficult to eradicate without catheter removal [21].

In the present study, 4 patients died of infection and 2 patients due to infection and progressive disease. Two patients died due to fungemia. Both patients were already on amphotericin B and the culture reports were available postmortem. In the case of fungal infections, catheter removal has strongly been recommended [13, 15, 23]. Another two patients died because of pneumonia. There was no catheter-related mortality. Others have reported similar experiences [3, 5, 15, 21].

Various risk factors have been shown to increase the risk of catheter-related infections. These include duration of catheterization, number of catheter manipulations, inexperience of the inserter, violation of aseptic techniques and triple-lumen catheters [7]. Other factors suggested to increase risk include neutropenia [5], young age [21] and hematological neoplasm [24]. A team approach, skin preparation with chlorhexidine and topical antibiotics have been found to reduce the risk of infection [7].

In conclusion, febrile neutropenia and catheter-related infectious episodes in neutropenic leukemia patients with long-term venous access in Kuwait are caused by both gram-positive and gram-negative organisms in equal frequency. The majority of the episodes are curable by antibiotic therapy without catheter removal.
40

Med Principles Pract 2000;9:35-41
Al Bahar/Pandita/Bavishi/Savani 


\section{References}

1 Press OW, Ramsey PG, Larson EB, Fefer A, Hickman RO: Hickman catheter infections in patients with malignancies. Medicine 1984;63: 189-200.

2 Larson EB, Wooding M, Hickman RO: Infectious complications of right atrial catheters used for venous access in patients receiving intensive chemotherapy. Surg Gynecol Obstet 1981;153:369-373.

3 Reed WP, Newman KA, Jongh CD, Wade JC, Schimpff SC, Wiernik PM, McLaughlin JS: Prolonged venous access for chemotherapy by means of the Hickman catheter. Cancer 1983;52:185-192.

4 Simon C, Suttorp M: Results of antibiotic treatment of Hickman-catheter-related infections in oncological patients. Support Care Cancer 1994; 2:66-70.

5 Howell PB, Walters PE, Donowitz GR, Farr BM: Risk factors for infection of adult patients with cancer who have tunnelled central venous catheters. Cancer 1995;75:13671375.

6 King DR, Komer M, Hoffman J: Broviac catheter sepsis: The natural history of an iatrogenic infection. J Pediatr Surg 1985;20:728-733.

7 Hampton AA, Sherertz RJ: Vascular access infections in hospitalized patients. Surg Clin North Am 1988;68: 57-71.

8 Blacklock HA, Hill RS, Clarke AG, Pillai MV, Matthews JRD, Wade JF: Use of modified subcutaneous right atrial catheter for venous access in leukaemic patients. Lancet 1980;i:993-994.

9 Lokich JJ, Bothe A Jr, Benotti P, Moore C: Complications and management of implanted vascualr access catheters. J Clin Oncol 1985;3 710-717.
10 Behre G, Link H, Maschmeyer G, Meyer P, Paaz U, Wilhelm M, Hiddemann W: Meropenem monotherapy versus combination therapy with ceftazidime and amikacin for empirical treatment of febrile neutropenic patients. Ann Hematol 1998;76:73-80.

11 Klastersky J: Science and pragmatism in the treatment and prevention of neutropenic infection. J Antimicrob Chemother 1998; 41(suppl D):13-24.

12 Keung YK, Watkins K, Chen SC, Groshen S, Silberman H, Douer D: Comparative study of infectious complications of different types of chronic central venous access devices. Cancer 1994;73:2832-2837.

13 Mueller BU, Skelton J, Callender DPE, Marshall D, Gress J, Longo D, Norton J, Rubin M, Venzon D, Pizzo PA: A prospective randomized trial comparing the infectious and non-infectious complications of an externalized catheter versus a subcutaneously implanted device in cancer patients. J Clin Oncol 1992; 10:1943-1948.

14 Alurkar S, Dhabhar BN, Pathak AB, Gonsalves I, Iyer RS, Kelker R, Pai SK, Deshpande RK, Desai PB, Advani SH: Longterm right atrial catheters in patients with malignancies: An Indian experience. J Surg Oncol 1992;51:183-187.

15 Riikonen P, Saarinen UM, Lahteenoja KM, Jalanko H: Management of indwelling central venous catheters in pediatric cancer patients with fever and neutropenia. Scand J Infect Dis 1993;25:357-364.

16 Krog MPM, Ekbom A, Rosander CN, Rudberg CR, Simonsson NOB: Central venous catheter in acute blood malignancies. Cancer 1987; 59:1358-1361.
17 Kappers-Klunne MC, Degener JE, Stijnen T, Abels J: Complications from long term indwelling central venous catheters in hematologic patients with special reference to infection. Cancer 1989;64:1747-1752.

18 Reilly JJ, Steed DL, Ritter PS: Indwelling venous access catheters in patients with acute leukemia. Cancer 1984;53:219-223.

19 Smith SR, Cheesbrough J, Spearing R, Davies JM: Randomized prospective study comparing vancomycin with teicoplanin in the treatment of infections associated with Hickman catheters. Antimicrob Agents Chemother 1989;33:1193-1197.

20 Lim SH, Smith MP, Salooja N, Machin SJ, Goldstone AH: A prospective randomized study of prophylactic teicoplanin to prevent early Hickman catheter related sepsis in patients receiving intensive chemotherapy for haematological malignancies. J Antimicrob Chemother 1991;28:109-116.

21 Benezra D, Kiehn TE, Gold JWM, Brown AE, Turnbull ADM, Armstrong D: Prospective study of infections in indwelling central venous catheters using quantitative blood cultures. Am J Med 1988;85:495498.

22 Hardman D, Englund R, Hanel K: Aspects of central venous access catheter usage in patients with malignancy. NZ Med J 1994;107:224226.

23 Raaf JH: Results from use of 826 vascular access devices in cancer patients. Cancer 1985;55:1312-1321.

24 Lazarus HM, Lowder JN, Herzig $\mathrm{RH}$ : Occlusion and infection in Broviac catheters during intensive cancer therapy. Cancer 1983;52:23422348 . 